\title{
DNS-Mikroarrays - Verbesserung der analytischen Plattformen zur Beschreibung molekularer Wirkmuster von Naturstoff- präparaten oder Science Fiction?
}

\author{
Oliver A. Wrulich, Florian Überall \\ Biozentrum Innsbruck, Abteilung für Medizinische Biochemie, Innsbruck, Österreich
}

\begin{abstract}
NS-Mikroarrays sind Träger aus Glas oder Kunststoff (Nylon), auf denen Erbmaterial in hoher Anzahl und Dichte in definierter Mikroanordnung (,Microarray") fixiert oder synthetisiert wird. Mikroarrays mit fixierten cDNS, Oligonukleotiden oder Fragmenten aus der Polymerasenketten Reaktionen nennt man gespottete Microarrays, solche wo direkt auf dem Biochip Oligonukleotide in hoher Auflösung synthetisiert werden, nennt man „High Density Oligonucleotide Microarrays (HDOs)".
\end{abstract}

\section{Der Biochip oder DNS-Mikroarray}

Das Messprinzip der DNS-MikroarrayAnalyse (Abb. 1) beruht auf der Wechselwirkung von Ribonukleinsäure (RNS)Molekülen mit ihren entsprechenden komplementären Desoxyribonukleinsäure (DNS)-Gegenstücken auf dem Biochip (DNS-Mikroarray). Das gewählte biologische Untersuchungsmaterial (z.B. menschliche Zellkulturen, Gewebefraktionen, Biopsien, Vollblut etc.) wird mit einem pflanzlichen Einzelstoff, Extrakt oder Vielstoffgemisch sowie dem korrespondierenden Lösungsmittel parallel behandelt. Anschliessend werden in einem ersten Schritt RNS-Moleküle aus dem behandelten Untersuchungsmaterial gewonnen und gereinigt. Nach einer Prozessierung der RNS in cRNS (komplementäre RNS) wird diese markiert und auf dem Biochip in räumliche Nähe zu DNS-Molekülen gebracht.

Die vollständige Sequenzierung des menschlichen Erbgutes bietet vielfältige Analysemöglichkeiten zur genomweiten Betrachtung aller Gene auf transkriptioneller, regulatorischer und funktioneller Ebene. Aufgrund der komplexen Zusammensetzung und der zu erwartenden multifaktoriellen Wirkungen von Naturstoffgemischen oder Phytopharmaka sind einer auf Evidenz basierten molekularen Wirkbeschreibung oftmals Grenzen gesetzt. Die DNS-Mikroarray-Technologie ist ein molekularbiologisches Hochdurchsatzverfahren, welches eine umfassende Analyse der Transkriptionsereignisse am Gesamtgenom erlaubt. DNS-Mikroarrays werden erfolgreich in vielen Bereichen der Zellbiologie zur Erfassung von differentiellen Genexpressionsereignissen eingesetzt und könnten auch neue Erkenntnisse zur Wirkungs- und Risiko-Nutzenabschätzung von pleiotrop wirksamen Naturstoffen und Lebensmitteln liefern. Die vorliegende Publikation befasst sich mit der Frage, inwieweit diese neue Technologie eine Verbesserung der analytischen Plattformen zur Wirkbeschreibung von Naturstoffpräparaten darstellt.

Schlüsselwörter: Bioinformatik, Genomik, Mikroarray, Naturstoffforschung, Sequenzierung, Systembiologie

\section{DNA-Microarray Technology - Useful for an Upgrading of Analytical Platforms for a better Understanding of Molecular Effects of Natural Compounds or just Science Fiction?}

The completed sequence data of the human genome offers a wide variety of methods to study all genes at the transcriptional, regulatory and functional levels in a genomewide manner. To identify the effects of mixtures of natural or herbal remedies, an evidence-based approach on the molecular level is often limited due to their complex composition and multifactorial effects. The DNA microarray technology is a highthroughput method which allows comprehensive analysis of transcriptional events on the overall genome. DNA microarrays are successfully used in many areas of cell biology for the detection of differential expressed genes and may also generate new insights into the impact and risk of pleiotropic active natural products and foods. The present paper addresses the question whether this new technology can be useful for an upgrading of the analytical platforms in order to improve the understanding of molecular effects induced by natural compounds in cellular test models and humans.

Key words: Bioinformatics, genomics, microarray, natural compounds, sequencing, systems biology

Im Falle passender komplementärer Gegenstücke an der DNS binden diese markierten RNS-Moleküle über Wasserstoffbrücken an die DNS (Abb. 2). Dieser Prozess wird Hybridisierung ge- nannt. Ungebundene oder unspezifisch gebundene RNS-Stücke werden abgewaschen und entfernt. In einem Scanner wird der Biochip ausgelesen, indem der Farbstoff durch Laserlicht an- 


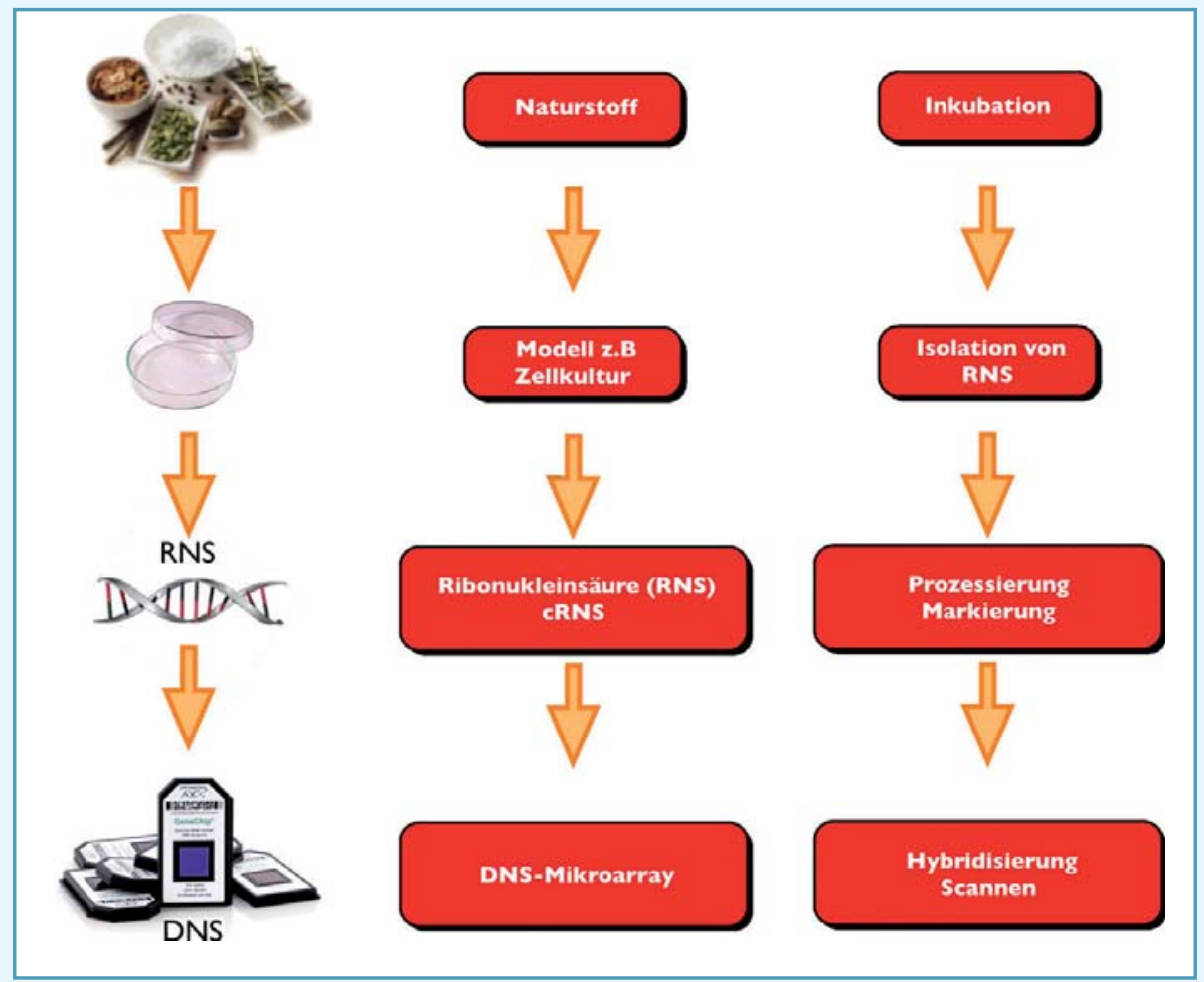

Abb. 1. Schematische Darstellung einer DNS-Mikroarray Analyse. Das Messprinzip beruht auf der Wechselwirkung von Ribonukleinsäure (RNS)-Molekülen mit ihren entsprechenden komplementären Desoxyribonukleinsäure (DNS)-Molekülen auf dem Biochip (DNS-Mikroarray). Das gewählte biologische Untersuchungsmaterial (hier menschliche Zellkultur) wird mit einem pflanzlichen Vielstoffgemisch sowie dem korrespondierenden Lösungsmittel parallel behandelt. Anschliessend werden in einem ersten Schritt RNS-Moleküle aus den behandelten Zellen gewonnen und gereinigt. Nach einer Prozessierung der RNS in cRNS (complementary (komplementäre) RNS) wird diese markiert und auf dem Biochip in räumliche Nähe zu den DNS-Molekülen gebracht. Nach erfolgter Bindung (Hybridisierung) und Laseranregung kann mittels CCD-Kamera die Fluoreszenzintensität ausgelesen werden. Sie ist ein indirektes Mass für die gebildete RNSMenge im Untersuchungsmaterial. Aus der Transkriptionsstärke kann die Genexpression abgeleitet werden. geregt und die Fluoreszenzintensität der verschiedenen Sektoren am Biochip quantifiziert wird. Die Fluoreszenzintensitäten, die mit den verschiedenen Bindeereignissen korrespondieren, liefern ein präzises Mass für die aus dem Untersuchungsmaterial isolierte RNS-Menge, welche auf dem Biochip mit DNS in Wechselwirkung getreten ist. Indirekt kann von der Höhe der Fluoreszenzintensitäten auf dem

Chip auf die Expressionsstärken geschlossen werden. Durch das Mikroarray-Rasterverfahren können alle differenziell regulierten Gene auf dem Biochip identifiziert und in eine Genliste übertragen werden. Diese Genlisten liefern dann die Grundlage für die bioinformatische Beschreibung der zellulären Wirkmuster in einer genomweiten systembiologischen Zusammenschau (Abb. 3).

Tab. 1. Auswahl von Genomgrössen sequenzierter Spezies. Die Genomgrösse ist in Megabasen (MB) angegeben.

$\begin{array}{cc}\text { Genomgrösse } & \text { Chromosomen } \\ 120 & 5 \\ 180 & 4 \\ 2.500 & 20 \\ 3.000 & 23 \\ 4.500 & 4\end{array}$

\section{Sequenzdatenbanken - Bibliotheken der Genome}

Methodische Weiterentwicklungen in der Sequenzierung der Erbsubstanz (DNS, Desoxyribonukleinsäure) haben zur vollständigen Entschlüsselung des genetischen Bauplanes verschiedenster Organismen geführt. Einem multinationalen Vorhaben (Humangenomprojekt) und einer privaten Initiative (Firma Celera) verdanken wir die ersten Entwürfe der Basensequenzen des menschlichen Erbgutes [1]. Am 29. April 2003, fünfzig Jahre nach der Aufklärung der DNS-Doppelhelix durch JAMES WATson und Francis Crick [2], wurde die Sequenz des menschlichen Referenzgenoms wesentlich vervollständigt und vom National Center for Biotechnology Information (NCBI) als „Version 33“ der gesamten wissenschaftlichen Welt online zur Verfügung gestellt [3]. Eine aktuelle Statistik (Januar 2009) zeigt im „Entrez Genome Project“ mit 834 komplett sequenzierten Genomen verschiedenster Organismen den Erfolg dieses Erweiterungsprogramms auf.

Im Vergleich einiger Organismen (Tab. 1) zeigt sich, dass die Komplexizität eines Organismus nicht mit der Genomgrösse korrelieren muss. So ist die Genomgrösse der Tabakpflanze mit 4.500 MB (Sequenzierung ist noch nicht abgeschlossen) deutlich grösser als die des Menschen (3.000 MB). Zusätzlich unterliegen etwa $74 \%$ aller MultiExon-Gene des menschlichen Genoms dem alterantiven Spleissen. Die Basensequenz eines Gens kann somit für unterschiedliche Proteine kodieren, wodurch z.B. eine menschliche Zelle mit rund 33.000 Genen viele hunderttausend verschiedene Proteine bilden kann. Aus relativ wenigen Genen kann dadurch ein ausserordentlich komplexes Proteom von 500.000 bis 1.000 .000 Proteinen entstehen, womit auch die intra-individuelle Variabilität des Genoms begründet ist [4]. Eine Sonderform des DNS-Mikroarrays, die ExonDNS-Mikroarray-Technik, berücksichtigt diesen Umstand (Abb. 4). Bei der Exon-Technologie wird jedes Gen in seiner Exon-Struktur analysiert und nicht nur ein oder zwei repräsentative Exons, wie beim herkömmlichen 3'-basierten Mikroarray. Damit ist es mög- 


\section{EXPRESSION}
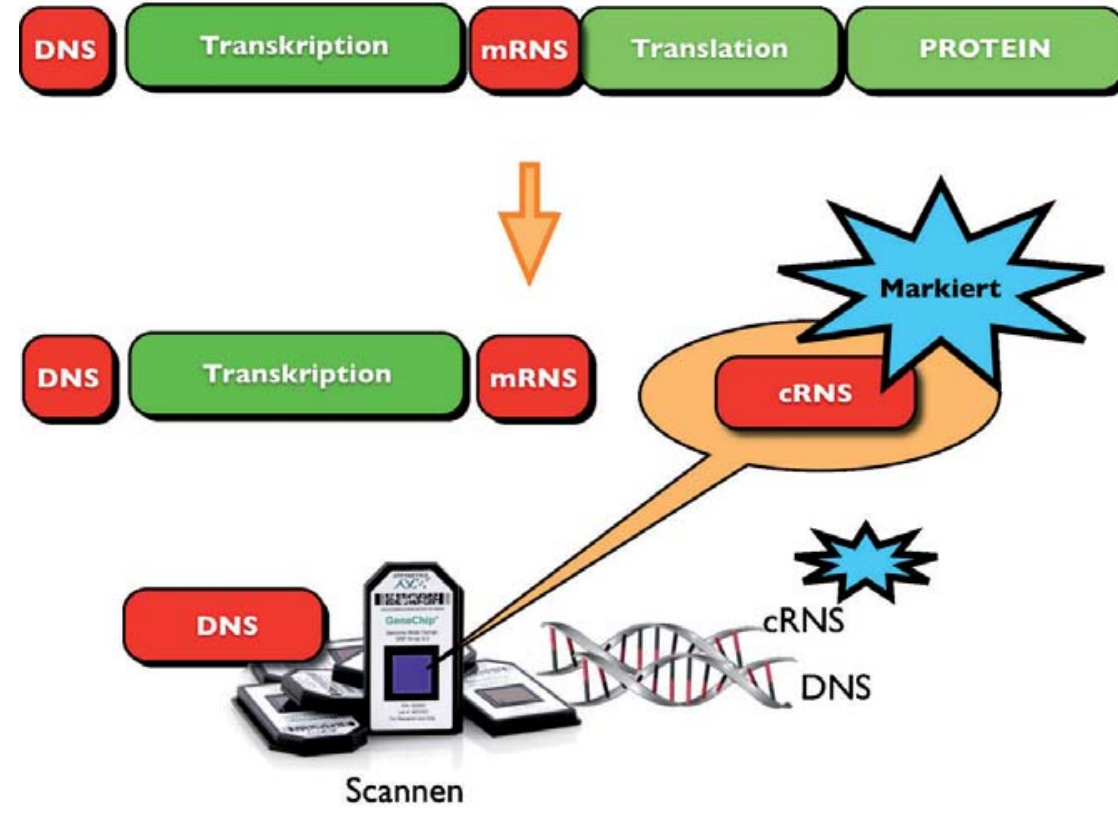

Transkriptomanalyse

Abb. 2. Transkription, Translation und Transkriptomanalyse. Transkription ist der Prozess des Umschreibens von genetischer Information aus Desoxyribonukleinsäuren (DNS) in Ribonukleinsäuren (RNS, Boten- oder messenger RNS, mRNS). Als Translation wird die Übersetzung der mRNSInformation in Proteine bezeichnet. Die Genexpression beschreibt die sequenzielle Abfolge von Transkription und Translation. Am Biochip kommt es zu einer sequenzspezifischen Wechselwirkung zwischen cRNS und DNS. Nach der Laseranregung kann die Fluoreszenzintensität ausgelesen und als sogenanntes CEL-File gespeichert werden. Eine DNS-Mikroarray-Analyse ist streng genommen eine Transkriptionsanalyse (Transcriptomics), da nur die Bildung von mRNS im Untersuchungsmaterial analysiert wird. Über die Bindungsaffinität zwischen RNS (aus dem Untersuchungsmaterial) und DNS am Chip kann man indirekt auf die Genexpression im Untersuchungsmaterial rückschliessen.

Genomischer Lokus

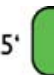

EXON

IN

INT

\section{EXON}

INT

T

EXON

EXON-Array
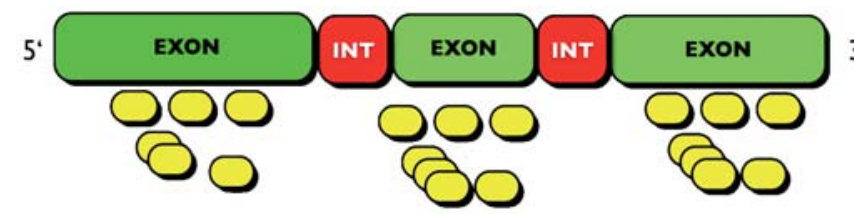

3'-Array

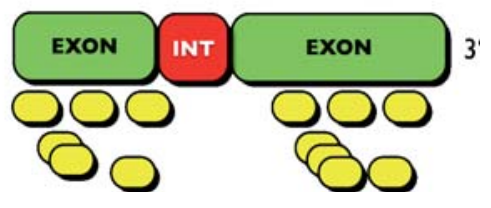

Abb. 3. Schematischer Aufbau des genomischen Lokus eines Gens (oben) mit Exons (grün) und Introns (rot), des EXON Arrays (Mitte) mit den Sonden (gelb) über alle Exons verteilt und (unten) der 3'-Array mit Sonden nur am 3'-Ende der ersten Exons. Da etwa 74\% aller Multi-Exon-Gene alternativ gespleisst werden, ermöglicht die Exon-Strategie auch die Analyse von Transkripten mit undefinierten 3'-Enden, nicht polyadenylierter RNS, degenerierten Proben, genomischen Deletionen und alternativen Spleissvarianten. lich Transkripte mit undefinierten 3 ' Enden, nicht polyadenylierte RNS, verstümmelte (trunkierte) Transkripte, alternative Polyadenylierungsstellen, degradierte Proben, genomische Deletionen und natürlich auch alternative Spleissvarianten, zu analysieren.

\section{Die Analyse der Gesamtheit}

Aus der systematischen Absicht, das gesamte Repertoire von exprimierten Genen eines Organismus zu erfassen, entstand der Begriff „Genomics“. In grossen Datenbanken wurden diese genomischen Informationen gespeichert und stehen für Sequenzvergleiche zur Verfügung. In den letzen Jahren sind zusätzliche Kunstwörter mit dem Suffix -omics entstanden. Diese omics-Suffixe bezeichnen Technologien, welche sich mit der Analyse von biologischen Funktionseinheiten beschäftigen. Die bekanntesten Technologiebereiche sind die Genomics (Analyse der Gesamtheit der Gene [Genom] eines Organismus), die Proteomics (Analyse der Gesamtheit der Proteine [Proteom] eines Organismus) und die Metabolomics (Analyse der Gesamtheit der Metaboliten [Metabolom/Metabonom] eines Organismus). Daneben gibt es noch Sonderformen, welche die besondere Anwendung der Genomik für Wirkbeschreibungen verdeutlichen wie z.B. die Phytopharmakogenomik (Wirkbeschreibung von Pflanzeninhaltsstoffen auf das Genom), die Nutrigenomik (Wirkbeschreibung von Nahrungsmitteln auf das Genom) oder die Toxikogenomik (Wirkbeschreibung von toxischen Substanzen auf das Genom).

Der Prozess der Namensfindung für solche Funktionskreise ist noch nicht abgeschlossen. Übergeordnete Funktionskreise sind ebenfalls denkbar. So kann man z.B. die Kombination der Genomik mit der Proteomik als Interaktomik bezeichnen. Allen omics-Technologien gemeinsam ist der Anspruch eines hohen Probendurchsatzes (engl. high throughput) bei der Analyse der Einzelelemente. Werden solche Verfahren, z.B. zum Auffinden neuer Tumortherapeutika und Medikamente aus grossen pflanzlichen oder synthetischen Stoffsammlungen verwendet, spricht 
man von high throughput screening (HTS).

\section{Transkriptionsanalyse als} Basis der Wirkbeschreibung

Präzise definiert ist die MikroarrayTechnologie ein DNS-DNS- oder DNSRNS-Nanoverfahren. Der Begriff der Genexpressionsanalyse hat sich etabliert, obwohl Transkriptionsereignisse analysiert werden. Mithilfe dieser Analysetechnik werden grundlegende physiologische und pathophysiologische Veränderungen in lebenden Zellsystemen, in Vollblut oder von Gewebebiopsien auf der Basis der gebildeten RNSMenge darstellbar. Diese Interpretation kann jedoch nicht direkt erfolgen, sondern erst nach Prozessierung der gewonnen RNS aus dem Untersuchungsmaterial (z.B. isolierte RNS aus Zellkulturen, Vollblut, Biopsien), Hybridisierung (Anlagerung von DNS an DNS oder cRNS an DNS auf dem Mikroarray) und Laserscanning. Bis $\mathrm{zu}$ einer endgültigen Aussage über die Änderung der Expression bedarf es aber einer aufwendigen statistischen und bioinformatischen Aufbereitung der Daten. Unabhängige Messanordnungen, z.B. Echtzeit-Polymerasekettenreaktion (RT-PCR), Western-Blot, siRNS-Knock-out (small interference RNS k.o.)-Experimente, Knock-out-Tiere (enthalten gentechnisch deaktivierte Gene) etc., werden dann zur Bestätigung der Expressionsereignisse herangezogen. Ein Vergleich von Transkriptionsprofilen liefert differentielle Expressionsunterschiede (Expressionsstärken) von hunderten bis tausenden Transkripten gleichzeitig zu Tage. Geht man hypothetisch davon aus, dass pflanzliche Gemische einen höheren Grad an multifaktoriellen Signalmustern erzeugen als pflanzliche oder synthetische Einzelsubstanzen, ist eine möglichst breite Informationsquelle zur Interpretation der Wirkmuster wünschenswert. Sinngemäss gilt die Abfragemöglichkeit mulifaktorieller Ereignisse uneingeschränkt auch für Einzelstoffe. Im Falle zu erwartender pleiotroper Wirkmuster pflanzlicher Gemische und Extrakte ist eine Hochdurchsatzplattform nötig. Die DNS-Mi-

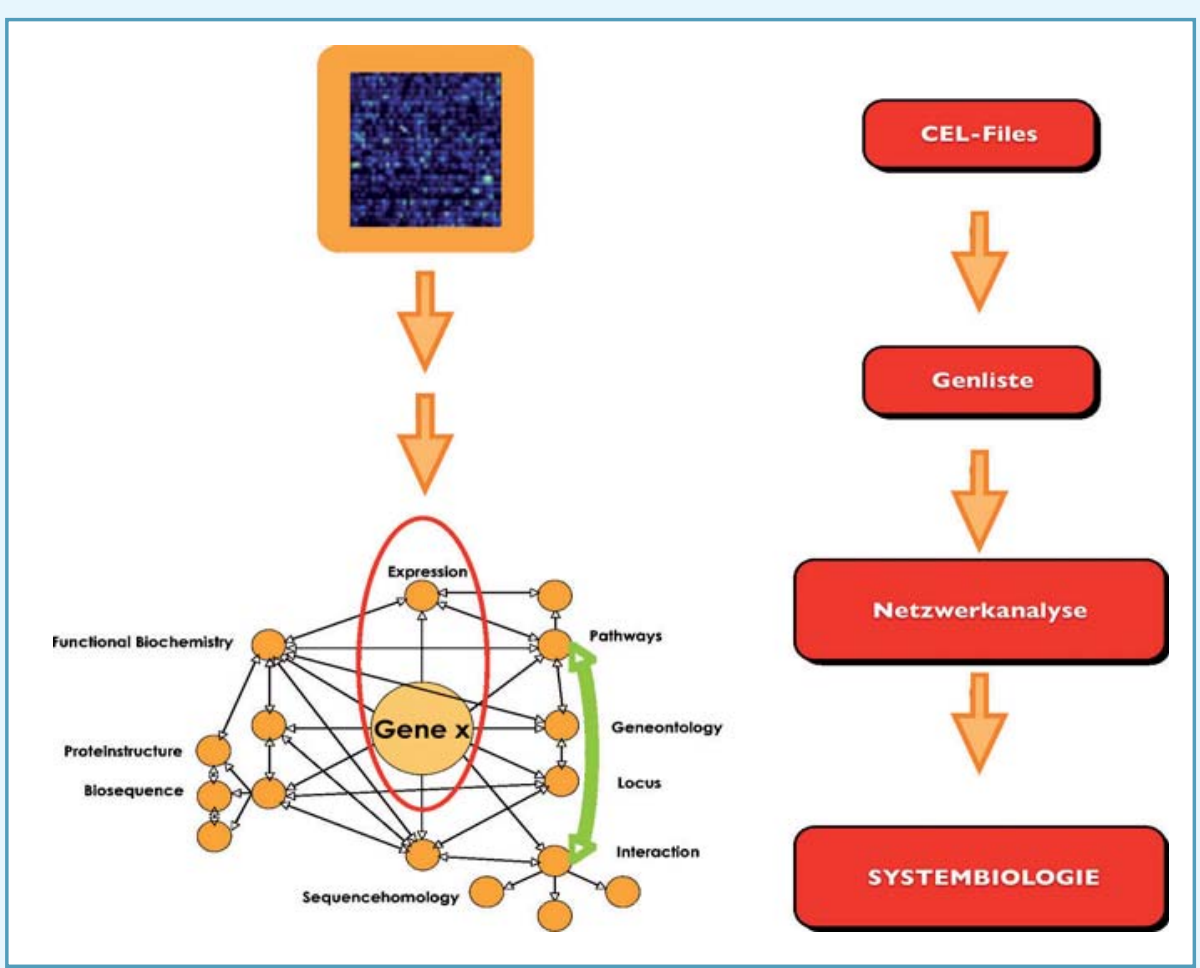

Abb. 4. Überführung der CEL-File-Information in die Genliste und von dort in eine funktionelle Netzwerkbeschreibung. In einem Scanner wird der Biochip ausgelesen, indem der Farbstoff durch Laserlicht angeregt und die Fluoreszenzintensität der verschiedenen Sektoren am Biochip quantifiziert wird. Die Fluoreszenzintensitäten, die mit den verschiedenen Bindeereignissen korrespondieren, liefern ein präzises Mass für die aus dem Untersuchungsmaterial isolierte RNSMenge, welche auf dem Biochip mit DNS in Wechselwirkung getreten ist. Indirekt kann von der Signalstärke (Fluoreszenzintensität) auf dem Chip auf die relative Expressionsstärke geschlossen werden. Durch das Mikroarray-Rasterverfahren können alle differenziell regulierten Gene auf dem Biochip identifiziert und in eine Genliste übertragen werden. Diese Genlisten liefern dann die Grundlage für die bioinformatische Netzwerkbeschreibung der zellulären Wirkmuster in einer genomweiten systembiologischen Zusammenschau.

kroarray-Technik liefert eine solche Plattform.

\section{DNS-Mikroarrays ... zur Stoffprüfung an Säugerkulturzellen}

Zur molekularen Analyse der Wirkbeziehungen von Naturstoffen benötigt man Modelle. Säugetierzellen in Kultur weisen im Vergleich zum menschlichen Organismus eine niedrigere biologische Komplexität auf. Dennoch sind Zellkulturen unschätzbare Hilfsmittel zur Beschreibung physiologischer Prozesse und ein anerkanntes Werkzeug der biomedizinischen Forschung zur Ermittlung reproduzierbarer biologischer Daten. Es gibt jedoch auch Einschränkungen bei der Beurteilung von DNS-Mikroarray-Daten aus ZellkulturExperimenten. Säugetierzellen in Kultur sind meist immortalisiert (durch
Einführung viraler oder zellulärer Onkogene unsterblich gemacht) und deshalb genetisch instabil. Immortalisierte Zellkulturen zeigen z.B. oft eine artifizielle Expression von Cytochrom P450-Isoenzymen, was zu Fehlinterpretationen von Metabolisierungsereignissen von Naturstoffen in Zellkulturen führen kann. Zellen ausserhalb des natürlichen Organverbandes benötigen Nährlösungen (Medien), die eine möglichst exakte Nachahmung der physiologischen Lebensbedingungen im Organverband gewährleisten. Diese Medien müssen neben Salzen, Vitaminen, Spurenelementen, Puffersubstanzen auch Wachstumsfaktoren, meist als tierisches fötales Serum, enthalten. Da die Zusammensetzung von Serum nicht konstant ist und mit dem Alter der Föten, deren Herkunft und Fütterung der Tiere variiert, sind die Versuchsergebnisse durch die nicht definierte und nicht konstante Zusammen- 
setzung der Seren oft nicht aussagekräftig oder vergleichbar. Weiters besteht bei tierischen und menschlichen Zellkulturen ein hohes Risiko einer Kontamination durch Bakterien, Pilze, Mycoplasmen und Viren aus Seren. Durch Testung der Serumchargen vor dem Einsatz in Zellkultur-Experimenten können aber nicht erwünschte Nebeneffekte vermieden werden.

Neben Säugertierzellkulturen werden häufig auch Hefestämme für Mikroarray-Untersuchungen eingesetzt. Zwei Publikationen, LOPEZ et al., 2008 [5], und Agarwal et al., 2008 [6], weisen Saccharomyes cerevisiae (Bäckerhefe) als gutes Hefemodell für genomweite Untersuchungen aus. Auf Grund der sehr gut verstanden Reproduktionsgenetik der Bäckerhefe eignet sich dieses System besonders gut für den HTS-Ansatz bei der genomweiten Wirkmusterfindung.

Im Umgang mit schlecht wasserlöslichen Naturstoffgemischen ist es wichtig zu beachten, dass Endkonzentrationen von $>1 \%$ organischem Lösungsmittel (z.B. Ethanol) im Kulturmedium bereits zu erheblichen Signalauslenkungen (z.B. Aktivierung von Kinasen) führen können. Neben der Verwendung diverser Zellkulturmodelle sind Primärkulturen (nicht immortalisierte Zellkulturen, welche direkt aus Gewebe gewonnen wurden) alternative Ausgangsmaterialien für Mikroarrays mit Naturstoffgemischen. Für beide Ansätze gilt das Konzentrations-EffektParadigma, das besagt, dass irrelevante Konzentrationsbereiche $\mathrm{zu}$ völlig unsinnigen und nicht interpretierbaren Daten führen können. Deshalb müssen Mikroarray-Experimenten immer Wachstumskinetiken und DosisWirkbestimmungen der Proben vorausgehen, welche eine Wirkung innerhalb vertretbarer biologischer Grenzen anzeigen. Dies gilt insbesondere für pflanzliche Vielstoffgemische schlechter Löslichkeit oder geringer Bioverfügbarkeit.

Beachtet man die zellbiologischen Einschränkungen durch Immortalisierung und Kulturbedingungen, sind Zellmodelle bei der Generierung von Wirkhypothesen jedoch eine gute Plattform für genomweite DNS-MikroarrayAnalysen mit Naturstoffgemischen.
Eine Aussage über physiologische Prozesse, wie sie im Zellverband stattfinden (z.B. Leberstoffwechsel), ist nur in Ausnahmemodellen (z.B. HeaptoSys Consortium, Analytik der Cytochrom P450-Isoenzyme in Mikrosomenfraktionen der Leber) möglich. Aber auch hier gelten Einschränkungen durch die gewählte CYP-Analytik.

\section{... zur Stoffprüfung an Tiermodellen}

Tiermodelle erweitern die Analyseplattform, um physiologische Verlaufsprozesse, wie z.B. die gastrointestinale Verdauung oder organspezifische Stoffaustauschmuster, untersuchen zu können. Auch hier gilt das KonzentrationsEffekt-Paradigma. Aussagen über die tatsächlichen bioaktiven Stoffkonzentrationen im jeweiligen Untersuchungsorgan sind aufgrund meist fehlender Aufnahmekinetiken und Metabolisierungsraten schwierig. Besonders hilfreich erscheinen Tiermodelle mit einem speziellen Funktionshintergrund (z.B. veränderter Hirnstoffwechsel, Arteriosklerose-, Stress-, Entzündungsmodelle etc.). Dieser Ansatz ist deshalb so wichtig, da Milieuänderungen zu veränderten metabolischen Ereignissen (z.B. Bildung von Sauerstoff- oder Stickstoffradikalen, Änderungen im Zytokinspiegel, sezernierte Botenstoffe, Wuchsstoffe etc.) führen, welche eine differentielle Genexpression auslösen können. Die Erfassung dieser milieubedingten Genexpression ist zur Beschreibung der systeminhärenten Auslenkungen vor der DNS-Mikroarray-Analyse wichtig. Erst damit werden physiologische und pathophysiologische Prozesse wechselseitig beschreibbar. Sie liefern die Daten für die Funktionsinterpretation der DNS-MikroarrayAnalyse und die Modellbildung.

Ein Beispiel zur Vertiefung dieser Ansicht zeigt das genomweite Expressionsprofiling mit einem standardisierten Pflanzenextrakt in einem RattenStressmodell. In diesem Modell [7] wurden männliche Sprague-Dawley-Ratten mit Johanniskraut-Extrakt STW3VI ${ }^{\circledR}$ und dem selektiven Serotonin-Wiederaufnahmehemmstoff Fluoxetin unter Stress (1h/Tag Restraint Stress für 21
Tage) behandelt. Untersucht wurde die Wirkung einer oralen Behandlung mit klinisch wirksamem Johanniskrautextrakt (STW3VI® ${ }^{\circledR}, 250$ und $500 \mathrm{mg} / \mathrm{kg}$ ) im Vergleich zu Fluoxetin ${ }^{\circledR}(10 \mathrm{mg} / \mathrm{kg})$ und Placebo. Neben neuroethologischen und biochemischen Parametern wurde eine DNS-Mikroarray-Analyse (Affymetrix Gene Chip Rat Genome 230 2.0) an Hypothalamus und HippocampusSchnitten der Ratten durchgeführt. Die Auswertung erfolgte mittels LIMMA (Linear Models for Microarray Analysis) und der PANTHER Datenbank. Erste Ergebnisse aus dem Hippocampus zeigen, dass chronischer Stress über 21 Tage zu einer differentiellen Regulation von 256 Genen im Vergleich zur ungestressten Kontrollgruppe führt. In der Fluoxetin-Gruppe konnten in gestressten Tieren 43 differentiell regulierte Gene gefunden werden. Die Gabe von STW zeigt konzentrationsabhängig $140(250 \mathrm{mg} / \mathrm{kg})$ bzw. 223 Gene (500 $\mathrm{mg} / \mathrm{kg}$ STW) differentiell reguliert. Einige der unter STW-Behandlung ausgelenkten Gene sind in Signalwegen repräsentiert, die hypothetisch an der Entstehung der Depression beteiligt sind. Eine Schnittmengenbildung jener Gene, die sowohl in die STW- als auch in die Fluoxetin-Gruppe fallen, ist nicht publiziert.

\section{... zur Beurteilung der Stoffwirkung im Menschen}

Zellkultur- und Tier-Modelle sind integraler Bestandteil zur molekularen Beurteilung biochemischer und physiologischer Prozesse. Die in diesen Modellen gewonnenen Daten können aber nicht unmittelbar auf den Menschen übertragen werden. Aus der Sicht der Patientensicherheit wäre es besonders wünschenswert, zwischen Respondern und Non-Respondern im Probandenkollektiv vor der Einnahme von Naturstoffen unterscheiden zu können. Dies setzt das Vorhandensein prädiktiver Genmuster voraus, die für diese personalisierte Diskriminierung der Wirkung verantwortlich sein könnten. In grossen placebokontrollierten Medikamentenstudien (z.B. Glivec ${ }^{\circledR}$ ) konnten solche Kollektive ansatzweise bestimmt werden. Einschränkend muss betont 
werden, dass diese Analytik an das Vollblut oder aus Vollblut isolierte und dann kultivierte periphere Monozyten (PBMCs, peripheral blood mononuclear cells) gebunden ist, da nur Zellpopulationen aus dem Blut für diese Analyse in Frage kommen.

\section{... zur Beschreibung molekularer Wirkmuster von Naturstoffen}

Im Wesentlichen gibt es zwei Hauptanwendungen von DNS-Mikroarrays in der Naturstoff-Forschung: (i) die Wirkmusterbeschreibung von pflanzlichen Einzelstoffen sowie (ii) die Testung und Wirkbeschreibung von Extrakten und komplex aufgebauten Vielstoffgemischen. Innerhalb der Naturstoffmedizin sind Mikroarrays prominent in den Feldern Phytopharmakogenomik, Phytopharmakogenetik und Nutrigenomik vertreten [8]. Zunehmend erobern DNS-Mikroarray-Techniken auch Felder der Zell- und Tumorbiologie. Besonders in der Tumorbiologie spielen DNS-Mikroarray-Techniken zur Erfassung von Genexpressionsänderungen eine besondere Rolle, da Gene, die in bestimmten Tumortypen erhöhte oder verminderte Expressionsraten aufweisen, als neue Marker in der Diagnostik angesehen werden.

In der Pharmakodynamik von Naturstoffen werden DNS-Mikroarrays zum Auffinden neuer diagnostischer und prognostischer Indikatoren und Biomarker der therapeutischen Wirkung sowie für die Aufklärung der molekularen Wirkmechanismen oder für die Identifizierung und Validierung neuer molekularer Angriffspunkte (z.B. Ionenkanäle, Rezeptoren) für die pflanzliche Arzneimittelentwicklung eingesetzt. In der Pharmakogenomik finden DNS-Mikroarrays Verwendung zur Vorhersage der Wirksamkeit, Sicherheit und Beschreibung potenzieller Nebenwirkungszenarien der pflanzlichen Naturstoffe bei präklinischen Studien. Sie sind wichtige Instrumente zur Identifizierung jener Gene, die Sensitivität und Resistenz gegenüber den verabreichten Naturstoffen auslösen.

Schliesslich sind DNS-Mikroarrays auch in der Pharmakognosie zur kor- rekten botanischen Identifizierung und nomenklatorischen Einordnung von pflanzlichen Rohstoffen als Teil der Standardisierung und Qualitätskontrolle von Nutzen. In zahlreichen Untersuchungen haben DNS-Mikroarrays bereits ihre Tauglichkeit für das genomweite Stoffaudit erfüllt. Ein Beispiel liefert die Arbeit von RoNG et al. [9]. Hier wurde die DNS-MikroarrayTechnologie für die Qualitätskontrolle von TCM-Produkten vorgeschlagen. Diese Form der genomischen HTSAnalyse soll, zusätzlich zu klassischen chromatographischen Methoden, sogenannte biologische „Fingerabdrücke“ (Genome-wide biological response fingerprints) der TCM-Formulierungen als Mass für die Qualität liefern.

Mit der erhöhten Nachfrage von wissenschaftlich validierten und standardisierten pflanzlichen Produkten steigt auch der Anspruch für ein besseres Verständnis der molekularen Mechanismen, welche der biologischen Wirkung von Naturstoffen zugrunde liegen.

Die Nützlichkeit von DNS-Mikroarrays bei der Identifizierung von Wirkprofilen pflanzlicher Vielstoffgemische soll exemplarisch anhand von Untersuchungen zu Ginkgo biloba-Blattextrakten beschrieben werden. Extrakte aus Blättern des ostasiatischen Baumes Ginkgo biloba werden seit Jahren als Nahrungsergänzungsmittel mit antioxidativer, neuroprotektiver und durchblutungsfördernder Wirkung, u.a. zur Verbesserung der Gedächtnisleistung, empfohlen. Obwohl die neuroprotektiven Effekte des standardisierten Ginkgo biloba-Extraktes EGb $761^{\circledR}$ auf molekularer und zellulärer Ebene sowie in Tiermodellen und Patientenstudien vielfach nachgewiesen wurde, blieb der molekulare Wirkmechanismus unbekannt. Diese Lücke konnte nun partiell mit DNS-Mikroarray-Experimenten geschlossen werden. Relevante neuromodulatorische Angriffspunkte konnten über die DNS-MikroarrayAnalyse identifiziert werden.

In einer Studie von Watanabe [10] wurden im Cortex und im Hippocampus von Mäusen, deren Nahrung über einen Monat mit EGb 761-Extrakt (300 $\mathrm{mg} / \mathrm{kg} ; \mathrm{n}=10$ ) ergänzt wurde, signifikant regulierte Gene gefunden, welche auf beschriebene neuromodulatorische Effekte hinweisen. Eine gleichgrosse Kontrollmausgruppe wurde mit dem Placebo behandelt. Aus den entnommenen Gewebeproben wurde RNS extrahiert, prozessiert und auf einem Maus Affymetrix MuU74 Gene Chip ${ }^{\circledR}$ Mikroarray hybridisiert. Von den insgesamt $\sim 6.000$ Genen und $\sim 6.000$ ESTs (expressed Sequence tags, EST's sind transkribierte Nukleotidsequenzen, die gewöhnlich durch Sequenzierung einer cDNS-Bibliothek erhalten werden) war nur ein Gen im Hippocampus und 9 Gene im Cortex mehr als 3-fach hochreguliert. Unter den 9 differentiell regulierten Genen im Cortex fanden sich Ionen-Kanäle, Wachstumshormone, Transkriptionsfaktoren und neuroprotektive Moleküle, deren Expression durch die Supplementierung mit EGb 761-Extrakt signifikant gesteigert wurde.

Ein ähnlicher experimenteller Aufbau, hier jedoch an einem PC12-Zellmodell, wurde von Sмітн et al. vorgestellt [11]. Mit EGb 761-Extrakt behandelte PC12-Zellen (Zellen stammen aus einem Nebennierentumor einer bestrahlten Ratte) konnten mehrere apoptose-regulierende Gene differenziell hinauf- bzw. hinunterreguliert aufgefunden werden. Die Autoren diskutieren die Hemmung apoptotischer Gene als jene gesuchten Signale, welche für die Neuroprotektion verantwortlich sind [11]. Weiterführende Experimente zur endgültigen Klärung der beschriebenen neuroprotektiven Signalwege unter EGb 761 stehen noch aus.

Eine kürzlich erschienene Publikation von Su et al. [12] verwendete erstmals einen Whole-Genome-DNS-Mikroarray (Mouse One Array ${ }^{\mathrm{TM}}$ ) mit 30.000 Genen, um die Effekte von EGb 761-Ginkgo-Extrakt auf die Genexpression im Frontallappen von Mäusen zu untersuchen, deren Nahrung für sieben Tage mit EGb 761-Extrakt (20 mg/kg) ergänzt wurde [12]. Von den insgesamt 29.922 analysierten Genen waren in dieser Studie 2209 Transkripte differentiell exprimiert, davon 1097 hoch- und 1112 hinunterreguliert.

Eine Pathwayanalyse mit diesen Daten zeigte, dass im Frontallappen der Maus 10 Signalwege statistisch signifi- 
kant $(p<0.05)$ mit der EGb 761-Behandlung assoziiert waren. Darunter fanden sich Signalwege mit hoher Präsenz in neurologischen Funktionsbereichen (,neuroactive ligand-receptor interaction pathway" $(\mathrm{p}=4.13 \mathrm{E}-06)$; „axon guidance pathway“ ( $\mathrm{p}=1.97 \mathrm{E}-$ 02); ,long-term potentiation pathway“ $(\mathrm{p}=3.34 \mathrm{E}-02)$.

Ein weiteres Beispiel der Brauchbarkeit der Mikroarray-Technik zur Aufklärung der molekularen Wirkung von Naturstoffen liefern Untersuchungen mit standardisierten, auf 5-Loxin angereicherten Weihrauch (Boswellia serrata)-Extrakten. Roy et al. [13] konnte dabei in einer DNS-MikroarrayAnalyse an menschlichen mikrovaskulären Endothelzellen (HMEC) vermutete anti-inflammatorische Effekte von Boswellia serrata-Extrakten auf transkriptioneller Ebene bestätigten [13]. Das proinflammatorische Zytokin TNF$\alpha$ induzierte in dieser Untersuchung 522 entzündungsrelevante Gene. Von diesen 522 Genen waren 113 Gene sensitiv auf eine Behandlung mit Boswellia serrata-Extrakten. Durch Zuhilfenahme von diversen Datenbanken (GenMAPP, KEGG) konnte gezeigt werden, dass besonders Matrix-Metalloproteinase-Gene von den BoswelliaExtrakten in der Expression gehemmt wurden. In unabhängigen Rückbestätigungsuntersuchen der Genexpression (mittels RT-PCR) konnte eine Sensitivität von Boswellia-Extrakten gegenüber VCAM-1 (vascular cell adhesion molecule-1, Ig-Superfamilie) und ICAM-1 (inter cellular adhesion molecule-1) bestätigt werden. In einem Carrageen-induzierbaren Ratten-Entzündungsmodell wurden ähnliche Daten gefunden.

Häufig führen Naturstoffe zu einer Stimulation oder Veränderung des Leberstoffwechsels. Ein interessantes DNS-Mikroarray-Modell zur Abfrage früher Toxizitätsprofile bei der Verabreichung von Lebertoxinen (drug-induced sensitivity studies) an Ratten und in der Nachbehandlung von Leberschnitten (rat liver slice model) wurde von ELFERINK et al. vorgestellt [14]. Da mehrere Zellsysteme (KupfferZellen, Stellatzellen und Hepatozyten) in einer multi-zellulären Biokonversion von Lebertoxinen beteiligt sind, haben die Autoren in diesen Experimenten versucht, ein Korrelat zwischen phenotypischen Leberschäden (Ratte) und Genexpressionsveränderungen (an $E x$ vivo-Gewebeschnitten) zu finden.

Die Autoren kommen zum Schluss, dass die aufgefundenen Genexpressionsmuster (Gene expression pattern) mit den phänotypischen Daten hoch korrelieren und das „Rat liver slice“Modell geeignet ist, um eine multizelluläre Lebertoxizität, auf der Basis des Genexpressionsprofilings, anzuzeigen. Diese Publikation und eine Arbeit von MinAMI et al. [15] zeigen die Brauchbarkeit der DNS-Mikroarray-Technologie zum Toxizitätsprofiling. Für Naturstoffe ergeben sich hier interessante Testmöglichkeiten zur Klärung lebertoxischer Eigenschaften.

In zahlreichen anderen Zellkulturmodellen wurden pflanzliche Extrakte, Auszüge oder Zubereitungen bei Genomstudien verwendet. So finden sich in der Literatur Hinweise auf eine DNSMikroarray-Analyse zur Wirkung von Genistein und PC-SPES (pflanzliche Mischung aus Scutellaria baicalensis, Glycyrrhiza glabra, Ganoderma lucidum, Isatis indigotica, Panax pseudoginseng, Dendranthema morifolium Rabdosia rebescens und Serenoa repens) in Prostata-Karzinomzellen [16], Übersichtsarbeit).

Heisswasserextrakte aus Pfingstrosenwurzeln (Paeonia radix) wurden in HepG2-Leberzellen mittels DNS-Mikroarray-Experiment untersucht. Die Rolle der differentiell regulierten pro-apoptotischen Gene blieb jedoch zweifelhaft. Coptidis rhizoma (Huanglian, TCM) und Berberin (Isochinolinalkaloid, z.B. aus der Berberitze) wurden an acht verschiedenen Pankreas-Krebslinien genomweit analysiert.

Ähnliche Studien gibt es zu Propolispräparaten und Echinacea bei Erkältungskrankeiten und zur verbesserten Wundheilung [16]. $\mathrm{Zu}$ den besonders umfassend beschriebenen pflanzlichen Monosubstanzen gehören Curcumin (Diferuloylmethan) und Resveratrol (3,5,4'-Trihydroxystilben [7]). Zahlreiche DNS-Mikroarray-Analysen beschreiben die antiapototische [18], proapoptotische [19] sowie antientzündliche Wirkung [20] von Curcumin.

In einer Zwischenbilanz kann man anmerken, dass DNS-Mikroarray-Ana- lysen in allen Bereichen der molekularen Naturstoff-Forschung anzutreffen sind. Die Einsatzgebiete reichen von einer genomweiten Wirkprofil-Erstellung bis hin zum Stoffaudit und zum Toxizitätsprofiling. Aufgrund genomweiter Abfragemöglichkeiten stellen DNS-Mikroarrays eine deutliche Erweiterung und Verbesserung der analytischen Plattformen zur molekularen Wirkmusterbeschreibung von Naturstoffen dar.

\section{Fazit}

DNS-Mikroarray-Technologien sind Hochdurchsatzverfahren, die auf der Wechselwirkung von RNS mit komplementären DNS-Molekülen beruhen. Die definierte Anordnung der DNS-Sonden am Biochip ermöglicht eine eindeutige Zuordnung der differentiell exprimierten Gene. Mit Hilfe statistischer und bioinformatischer Methoden kann dann aus der Fluoreszenzintensität (Signalstärke) auf dem Biochip das Expressionsverhalten ausgelesen werden. In Ergänzung zu massenspektrometrischen Methoden (MALDI-TOF, SELDITOF etc.) zur Verlaufskontrolle der unter dem Einfluss von Naturstoffen freigesetzten Biomarker erweitert die DNS-Mikroarray-Technologie die bestehende Analytik auf das gesamte Genom ausgewählter Untersuchungsmodelle.

Zur Sicherung der Ansprüche von Unbedenklichkeit und Wirksamkeit von Naturstoffpräparaten sind robuste kostengünstige Hochdurchsatzverfahren anzustreben.

Für die Bewertung pleiotrop wirkender Naturstoffgemische sind genomweite Expressionsprofile hilfreiche Analysemethoden zur Klärung physiologischer oder pathophysiologischer Funktionen im gewählten Untersuchungsmaterial. Eine molekulare Risiko-Nutzenabschätzung von bioaktiven Vielstoffgemischen muss sowohl die allgemein gültigen Anforderungen an Phytoarzneimittel erfüllen, aber auch die Besonderheiten der pleiotropen Wirkung angemessen berücksichtigen [21]. In Zellkulturen oder Tiermodellen sind solche Risiko-Nutzenabschätzungen methodisch leichter durchführbar, 
was zu einer breiten Anwendung der DNS-Mikroarray-Technologien in Zellmodellen geführt hat.

Der unmittelbare Nutzen von DNSMikroarrays zur Beurteilung der Wirkung oder eines Risikopotentials beim Menschen nach Einnahme von Naturstoffpräparaten ist vorerst auf das Vollblut beschränkt. Die flächendeckende Entnahme von Vollblut im Zuge präklinischer und klinischer Prüfungen werden jedoch die Anwendungshäufigkeit der DNS-Mikroarray-Plattformen zur molekularen Beschreibung der Naturstoffwirkungen vergrössern. Die Überwindung bestehender technischer Schwachstellen, neue kostengünstigere Biochip-Lösungen sowie verbesserte Algorithmen zur Auswertung werden ihren Teil zur Implementierung von DNS-Mikroarray-Plattformen für die breite Naturstoff-Forschung beitragen. Gemeinsam mit Techniken der Proteomik, Metabolomik und Massenspektrometrie werden genomische Verfahren in den nächsten Jahren zu wichtigen Instrumenten der Naturstoff-Forschung werden.

\section{Danksagung}

Für die Durchsicht des Manuskriptes und wertvolle Literaturrecherchen möchte ich Dr. Marcel Jenny in meiner Arbeitsgruppe besonders herzlich danken.

\section{Literatur}

1. http://www.celera.com (29.01.2009)

2. Watson JD and Crick FHC: Molecular Structure of nucleic acids: a structure for deoxyribose nucleic acid. Nature 1953;4356: 737-738.
3. http://www.ncbi.nlm.nih.gov/genomes/static/gpstat.html (13.01.2009)

4. Schwabl H und Vennos C: Systems Biology Herausforderung und Anregung für die Komplementärmedizin. Schweiz. Zschr. GanzheitsMedizin 2008;20:360-364.

5. Lopez A, Parsons AB, Niskow C, Giaever G, Bonne C: Chemical-gentic approaches for exploring the mode of action of natural products. Prog Drug Res. 2008;337:239-71.

6. Agarwal AK, Xu T, Jacob MR, Feng Q, Li XC, Walker LA, Clark AM: Genomic and genetic approaches for the identification of antifungal drug targets. Infect Disor Drug Targets 2008; 1:2-15.

7. Jungke P, Ostrow G, Li J, Kolb C, Kelber O, Nieber K, Butterweck V: Genexpression in Hypothalamus und Hippocampus nach Behandlung mit Johanniskraut (STW 3-VI) oder Fluotexin in einem Modell für chronischen Stress. Zeitschrift für Phytotherapie 2009:S 01 (Vorträge).

8. Ulrich-Merzenich G, Zeitler H, Jobst D, Panek D. Vetter $\mathrm{H}$, Wagner $\mathrm{H}$ : Application of the "-Omic-" technologies in phytomedicine. Phytomedicine 2007;14:70-82.

9. Rong J, Tilton R, Shen J, Ng KM, Liu C, Tam PK, Lau AS, Cheng YC: Genome-wide biological response fingerprinting (BioReF) of the Chinese botanical formulation ISF-1 enables the selection of multiple marker genes as a potential metric for quality control. J. of Ethnopharmacology 2007;113:35-44.

10. Watanabe CM, Wolffram S, Ader P, Rimbach G, Packer L, Maguire, Schultz PG, Gohil K.: The in vivo neuromodulatory effects of the herbal medicine Ginkgo biloba. Proc Natl Acad Sci USA 2001:98:6577-6580.

11. Smith JV, Burdick AJ, Golik P, Khan I, Wallace D, Luo Y: Anti-apoptotic properties of Ginkgo biloba extract EGb 761 in differentiated PC12 cells. Cell Mol Biol 2002;48:699-707.

12. Su SY, Hsieh CL, Wu SL, Cheng WY, Li CC Lo HY, Ho TY, Hsiang CY: Transcriptomic analysis of EGb 761-regulated neuroactive receptor pathway in vivo. J Ethnopharmacol. 2009:4:123(1):68-73.

13. Roy S, Khanna S, Shah H, Rink C, Phillips C, Preuss $H$, Subbaraju GV, Trimurtulu G', Krishnaraju AV, Bagchi M, Bagchi D, Sen CK: Human genome screen to identify the genetic basis of the anti-inflammatory effects of Boswellia in microvascular endothelial cells. DNA Cell Biol. 2005;4:244-55.

14. Elferink MG, Olinga P, Draaisma AL, Merema MT, Bauerschmidt S, Polman J, Schoonen WG, Groothuis GM: Microarray analysis in rat liver slices correctly predicts in vivo hepatotoxicity. Toxicol Appl Pharmacol 2008;229: 300-9.

15. Minami $K$, Saito $T$, Narahara $M$, Tomita $H$ Kato H, Sugiyama H, Katoh M, Nakajima M, Yokoi T: Relationship between hepatic gene expression profiles and hepatototoxicity in five typical hepatotoxicant-administered rats. Toxicological Sciences 2005;87:296-305.

16. Hudson J, Altamirano M: The application of DNA micro-arrays (gene arrays) to the study of herbal medicines. J. Ethnopharmacology. 2006;108:2-15.

17. Bauer JA, Sinclair DA: Therapeutic potential of resveratrol: the in vivo evidence. Nature Reviews Drug Discovery, 2006;5:493-506.

18. Skommer J, Wlodkowic D, Pelkonen J: Geneexpression profiling during curcumin-induced apoptosis reveals downregulation of CXCR4. Exp. Hematol. 2007;35:84-95.

19. Yan C Jamaluddin MS, Aggarwal B, Myers J Boyd DD: Gene expression profiling identifies activating transcription factor 3 as a novel contributor to the proapoptotic effect of curcumin. Molecular Cancer Therapeutics. 2005; 5:233-241.

20. Teiten $M H$, Eifes $S$, Reuter $S$, Duvoix $A$ Dicato A, Diederich M: Gene expression profiling related to anti-inflammatory properties of curcumin in K562 leukemia cells. Annals of the New York Academy of Sciences. 2009; 1171:391-398.

21. Wrulich OA, Jenny M, Überall F: Molekulare Risiko-Nutzenbewertung von Phytopharmaka: Neue Herausforderungen oder unerfüllbare Vorgaben? Phytotherapie 2009;2/09:7-13.

\section{Disclosure Statement}

The author declares that no financial or other conflict of interest exists in relation to the content of this article.

\section{Korrespondenzadresse}

A. Univ. Prof. Mag. Dr. Florian Überall, PhD Biocenter Innsbruck

Division of Medical Biochemistry

Functional Gene Expression Bioinformatics Nutrigenomics Unit

Innsbruck Medical School

Fritz Preglstr. 3, A-6020 Innsbruck

florian.ueberall@i-med.ac.at 